\title{
Investigation of shock waves in explosive blasts using fibre optic pressure sensors
}

\author{
S Watson ${ }^{1}$, W N MacPherson ${ }^{1}$, J S Barton ${ }^{1,5}$, J D C Jones ${ }^{1}$, A Tyas ${ }^{2}$, A V Pichugin ${ }^{2}$, A Hindle², W \\ Parkes $^{3}, C$ Dunare ${ }^{3}$ and T Stevenson ${ }^{3}$ \\ ${ }^{1}$ School of Engineering and Physical Sciences, Heriot-Watt University, Edinburgh EH14 4AS, UK \\ ${ }^{2}$ Department of Civil \& Structural Engineering, University of Sheffield, Sheffield S1 3JD, UK \\ ${ }^{3}$ Scottish Microelectronics Centre, Kings Buildings, West Mains Road, Edinburgh EH9 3JF, UK
}

\begin{abstract}
We describe miniature all-optical pressure sensors, fabricated by wafer etching techniques, less than $1 \mathrm{~mm}^{2}$ in overall cross-section with rise times in the $\mu$ s regime and pressure ranges typically $900 \mathrm{kPa}(9 \mathrm{bar})$. Their performance is suitable for experimental studies of the pressure-time history for test models exposed to shocks initiated by an explosive charge. The small size and fast response of the sensors promises higher quality data than has been previously available from conventional electrical sensors, with potential improvements to numerical models of blast effects. Results from blast tests are presented in which up to 6 sensors were multiplexed, embedded within test models in a range of orientations relative to the shock front.
\end{abstract}

Keywords: blast wave, explosive charge, shock, fibre optic sensor, pressure

Short title: Explosive blast measurement

\section{Introduction}

Explosive blast wave research is all too relevant to safety and security in the modern world. In order to design buildings and structures which mitigate the effects of explosive blasts, we need accurate models of the pressure-time history from which the transient forces on the structures can be calculated. Modelling the behaviour of blast waves propagating into a region including obstructions remains one of the most challenging problems in engineering. For example, there are still uncertainties in realistic modelling of the real gas effects, especially in the vicinity of an explosion. Numerical modelling of blast waves is quite advanced nowadays, but accurate tracking of shocks across tens and hundreds of metres, which are typical distances for the scale relevant to a civil engineer, requires enormous computational costs. As a result, it is often necessary to resort to experimental testing, sometimes at the full scale, which can be difficult, dangerous and expensive. It is therefore tempting to consider small scale testing. However, surprisingly little is known about the applicability of scaling results from small scale trials to predict full scale effects $[1,2]$. For example, the "ConWep" software [3], whose predictions are based on the extensive tests by the American military [4], is known to predict successfully the parameters of blast waves produced by relatively large explosions, but the lower applicability limit is not well specified. This problem motivated our research into the practical small scale investigation into the parameters of blast waves reflected by relatively simple rectangular structures. If the scaling laws can be verified experimentally for explosive charges of the order of tens of grams, it is possible to envisage blast wave propagation experiments on scale models of complex geometries such as city streets, analogous to scaled wind tunnel experiments, to test the validity of numerical codes beyond their present limits.

Pressure (side-on or reflected) is a very useful parameter of the blast wave; however, it is very difficult to measure accurately, and high bandwidth resolution is required to measure the impulse force. An ideal sensor for the task would have to be sufficiently robust to withstand a blast loading; small to reduce interaction with the flow; possess a high natural frequency with suitable damping to record the pressure-time history accurately, and be insensitive to noise, both electromagnetic and mechanical, produced by the blast. While each of these requirements may be addressed separately with relative ease, no truly comprehensive solution has been presented so far. For example, commercially available specialised piezo-electric sensors are very robust with working pressures of hundreds of bar and rise times on the microsecond scale. Nevertheless, they are typically expensive, bulky ( $\sim 1 \mathrm{~cm}$ in diameter), and

\footnotetext{
${ }^{5}$ To whom correspondence should be addressed: J.S.Barton@hw.ac.uk
} 
susceptible to acceleration, which makes accurate pressure measurements nearly impossible. Small scale experiments impose additional restrictions on size of the sensing area, sensor body and embedding requirements, especially when it is necessary to measure flow details near sharp edges of the test models.

A number of optical fibre pressure sensors have been reported in the literature, the majority of which are interferometric [5-11]. In general, these are short-cavity Fabry-Perot sensors formed by bonding a thin flexible diaphragm to a supporting structure attached to the end of an optical fibre and interrogated in reflection. Provided the reflectivities of the fibre and diaphragm are low, the Fabry-Perot cavity is low finesse and the intensity of the reflected signal follows an approximately sinusoidal response as the cavity length changes in response to external pressure changes. The optical phase change can be inferred by using single or multiple wavelength sources, or by white light interferometry. Assuming a uniform thickness circular diaphragm, the sensor performance can be optimised for a particular application by choosing the diaphragm's material properties (density, Young's modulus and Poisson's ratio) and size (thickness $h$ and radius $a$ ). For a given material, the phase sensitivity to an external pressure change scales as $a^{4} \cdot h^{-3}$ and the lowest resonant frequency scales as $h \cdot a^{-2}$ [12].

Such optical fibre pressure sensors have several potential advantages that are pertinent to blast wave experiments. Their small size results in good spatial resolution and high packing density for mapping the pressure distribution over the surface of a test model. The low mass diaphragm is relatively insensitive to acceleration and, with resonant frequencies typically above $1 \mathrm{MHz}$, enable data capture over a bandwidth exceeding $100 \mathrm{kHz}$. The mainly dielectric construction provides immunity to electromagnetic interference and pickup, and electrical connections are not required. The manufacture of sensor bodies by silicon wafer processing techniques is expected to lead to a low cost per sensor. We have already demonstrated the potential of earlier versions of these sensors in transient aerodynamic experiments [13], in which 5 sensors were embedded in the trailing edge of a nozzle guide vane in a turbine test rig to measure the unsteady pressure upstream of the rotor. Pressure fluctuations of $90 \mathrm{kPa}(100 \mathrm{kPa}=1 \mathrm{bar})$ were measured at the $8 \mathrm{kHz}$ rotor blade passing frequency with a resolution of $0.65 \mathrm{kPa}$, with harmonics of up to $180 \mathrm{kHz}$.

In this paper we describe experiments to compare fibre optic pressure sensors with conventional gauges, to measure the loads on the faces of structures explosive blasts in small scale testing and to investigate the applicability of existing scaling data. Our results are illustrated by the data from representative trials.

\section{Operating principle}

Reference [13] describes the sensors and the associated optical system, so we restrict the description here to an outline only. Fabry-Perot cavities were formed between a cleaved fibre end and a flexible diaphragm at the end of a micro-machined sensor cavity, typically $20-100 \mu \mathrm{m}$ long and sealed at atmospheric pressure (figure 1). The low reflectivities of the fibre end and diaphragm resulted in an approximately sinusoidal response of the reflected optical signal versus external pressure and, for small deflections of the diaphragm, the optical phase was expected to vary linearly with pressure change. The fibres were singlemode at $1550 \mathrm{~nm}$ and the sensors were interrogated with high stability $(<1 \mathrm{pm} / 24 \mathrm{hr}) 10 \mathrm{~mW}$ fibre pigtailed DFB laser diode sources at 1532.3, 1546.5 and $1562.6 \mathrm{~nm}$ packaged in a commercial system (Profile 8000). The three resulting reflection signals follow three sinusoidal response functions as pressure changed, the phase separation of which was set by the source wavelengths. Phase was overdetermined by the three measurements and was thus insensitive to common mode intensity noise and changes in the visibility of the interference [12]. This interrogation method was chosen because the optical sources were unmodulated, unlike time-division multiplexed methods, allowing data capture over a high signal bandwidth, in principle only limited by the rise time of the detectors and associated amplifiers. A Labview program was written to display wrapped phase (i.e. modulo $2 \pi$ ) on-line for rapid evaluation of data quality, and further post-processing using Matlab generated the unwrapped phase and applied the sensor calibrations to give the pressure - time signals. The optical detection system (figure 1) was all-fibre in construction, using fibre Bragg gratings (Lumen Photonics) as wavelength filters for the 1546.5 and $1562.6 \mathrm{~nm}$ signals [13]. Grating reflectivities exceeded $99 \%$ with spectral widths greater than $1 \mathrm{~nm}$ FWHM. The lasers were capable of addressing 6 sensors simultaneously through a fibre coupler tree with a detection system required for each sensor. Three high speed $(<0.2 \mathrm{~ns}$ rise time) InGaAs pigtailed photodetectors (Hamamatsu) were used to detect the three wavelengths in each sensor channel. The detection bandwidth was $500 \mathrm{kHz}$ at $-3 \mathrm{~dB}$, set by the post-detector amplifiers. The optical system used fibre connectors which eliminated the need for splicing equipment and facilitated setting up on site. 


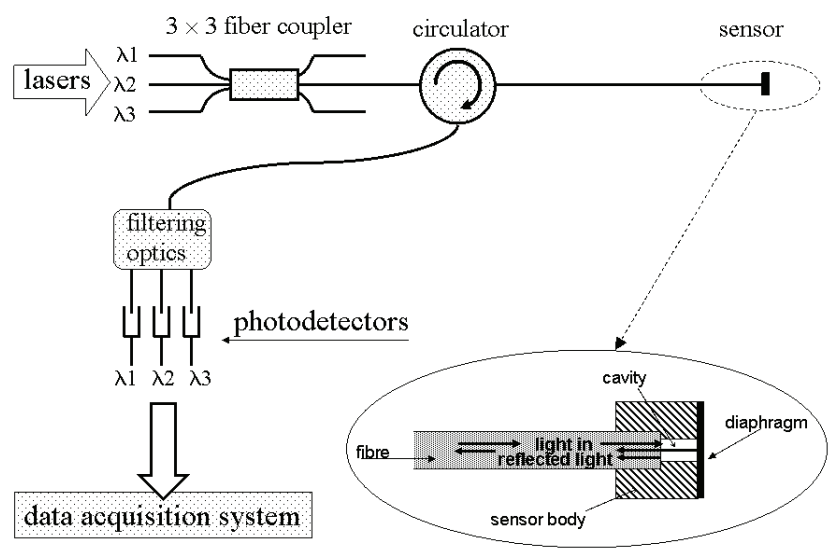

Figure 1. Principle of operation of sensors and optical interrogation system.

\section{Sensor fabrication}

The sensor bodies were fabricated on a 3 inch diameter, $380 \mu \mathrm{m}$ thick silicon wafer. Deep etching by the Bosch process [14] was used to create the fibre holes to a depth of approximately $360 \mu \mathrm{m}$ on the front side. On the back face, a similar pattern of smaller holes to define the pressure sensing apertures was aligned to the front array. The sensor cavity holes of various diameters were deep etched through the remaining $20 \mu \mathrm{m}$ depth to the large holes in the front side (figure 2). A $1 \mu \mathrm{m}$ thick silicon dioxide $\left(\mathrm{SiO}_{2}\right)$ membrane was grown thermally on an auxiliary 3 -inch silicon handle and transferred to the device wafer by a bonding technique.

To transfer the $\mathrm{SiO}_{2}$ layer to the etched device wafer, the oxide film on the handle wafer was brought into contact with the array of pressure sensing holes on the other wafer so that the surfaces would adhere together by weak bonding forces. The wafers were then annealed to strengthen the bonds considerably. Finally, a rapid inductively-coupled plasma silicon dry etch process was used to remove the handle wafer, leaving the $\mathrm{SiO}_{2}$ membrane intact and firmly bonded to the rear surface of the device wafer (figure 3).

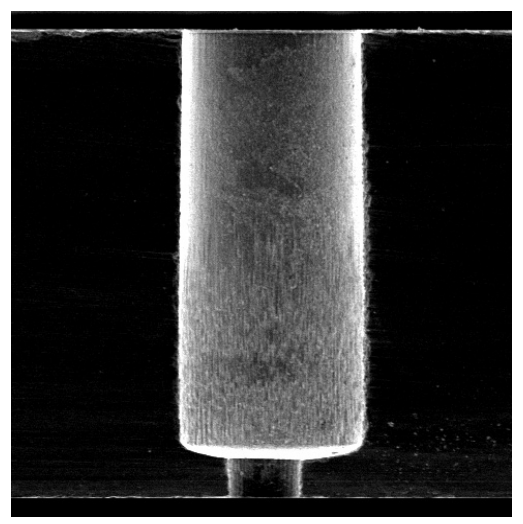

Figure 2. Scanning electron microscope crosssection of a portion of the device wafer $380 \mu \mathrm{m}$ thick, showing the fibre entry hole of diameter $128 \mu \mathrm{m}$ above the $50 \mu \mathrm{m}$ diameter sensing cavity.

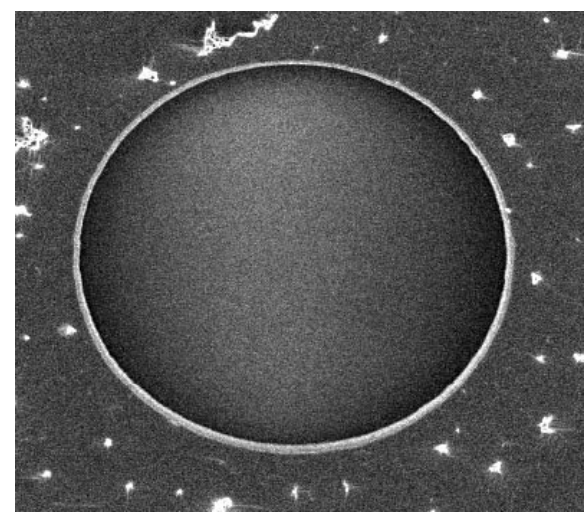

Figure 3. SEM image of the $\mathrm{SiO}_{2}$ membrane covering one of the $50 \mu \mathrm{m}$ diameter cavities, showing the internal silicon step surrounding the hole at the bottom of the fibre channel.

The wafers were diced and assembled into discrete devices. The diced parts, termed sensor bodies, typically had an area of approximately $1 \mathrm{~mm}^{2}$ with nominal diaphragm diameters of 50,75 and $100 \mu \mathrm{m}$. These were inspected under a microscope to ensure that there were no known imperfections before proceeding with assembly into a sensor. Their diaphragm surfaces, within the cavity, were then made 
reflective by coating with between 10 and $100 \mathrm{~nm}$ of aluminium. This was achieved by positioning them in a vacuum coating chamber with their fibre entry holes facing the thermal evaporation source.

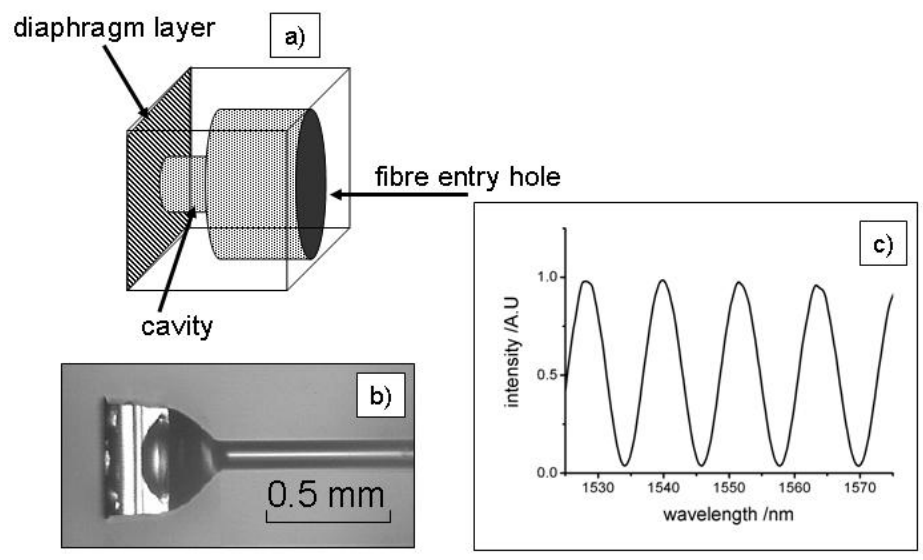

Figure 4. (a) Illustration of sensor body. (b) Photograph of assembled sensor.

(c) Interference fringes produced by illuminating a sensor with a broadband source.

Figure $4 \mathrm{a}$ illustrates the design of a sensor body. The assembly of a sensor involved the operator guiding a $125 \mu \mathrm{m}$ diameter cleaved fibre into the fibre entry hole, with the aid of a microscope and xyz translation stage. The other end of the fibre was coupled to both a broadband source and an optical spectrum analyser by a 50/50 fibre coupler. This enabled the operator to observe the appearance of fringes from the formation of the Fabry-Perot cavity as the fibre was inserted. When satisfactory fringes appeared and the fibre was butted against the step before the cavity, epoxy was applied between the fibre and the outside of the sensor body to fix the fibre in place and seal the cavity. Figures $4 \mathrm{~b}$ and $4 \mathrm{c}$ show an assembled sensor and the fringe pattern of the sensor cavity. The free spectral range measured from the fringe pattern was used to calculate the length of the cavity. This provided the measure of the phase steps between the laser sources, with respect to the sensor transfer function, which were necessary for the algorithm [15] used to calculate the interferometric phase.

\section{Sensor calibration and mounting}

Sensors were tested for their response to pressure changes by installing them in a pressure chamber for calibration or a small shock tube for dynamic testing. They were repeatedly cycled through pressure changes from atmospheric pressure to an absolute pressure of approximately 9 bar (figure 5 ) to calibrate their response (lower curve) with respect to a low-bandwidth electrical sensor (upper curve). A plot of phase against pressure for a $50 \mu \mathrm{m}$ diameter diaphragm sensor (figure 6) shows no significant departure from a linear fit to the phase response across the operating range, and no discernable hysteresis. Phase

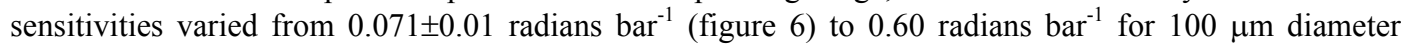
sensors. The final test for each sensor was to expose it to a face-on air shock of typically 2 to 3 bar in the shock tube (figure 7), to confirm its suitability for explosive blast experiments. The shock tube consisted of two $1 \mathrm{~m}$ lengths of $25 \mathrm{~mm}$ inside diameter pipe separated by a Mylar burst-diaphragm. The working section side of the tube was filled with air, and the driver side was pressurised from a nitrogen supply to reach the diaphragm bursting pressure. The $10 \%-90 \%$ rise time of the signal at the shock in figure 7 is $1.1 \mu \mathrm{s}$. 

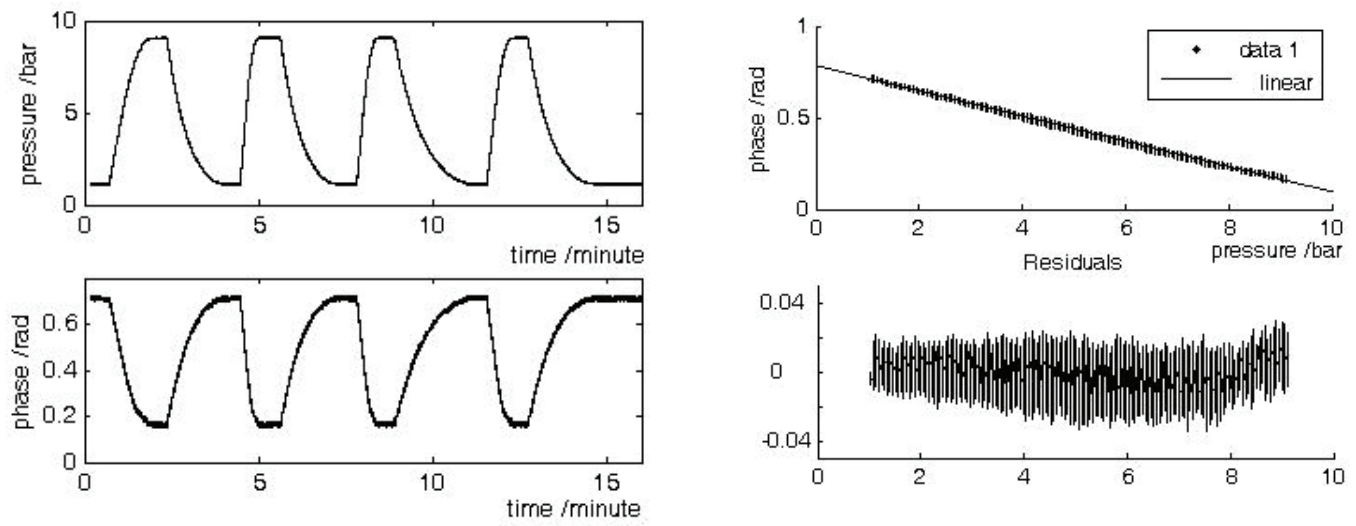

Figure 5. Pressure cycling experiment. Electrical reference sensor (upper curve); optical sensor phase signal (lower curve)

Figure 6. Optical sensor calibration derived from Figure 5. Lower plot shows the residuals to a linear phase - pressure fit.

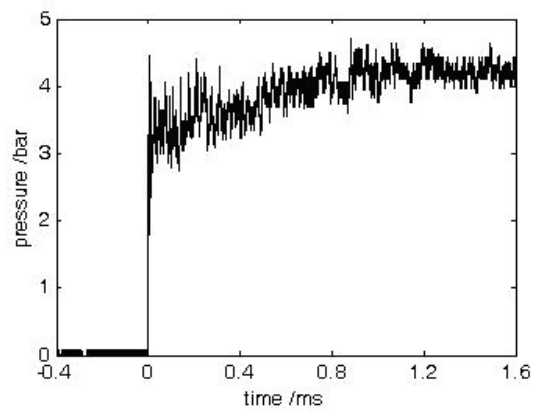

Figure 7. Optical sensor signal in response to a normal incidence shock.

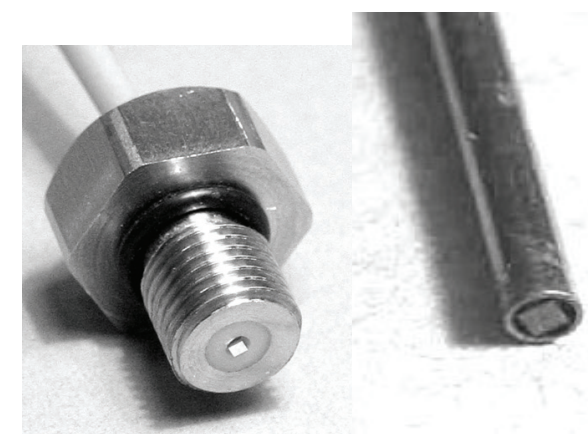

Figure 8. Mounted optical fibre sensors: (left) HKM-375 style mounting; (right) XCQ-080 style mounting.

Our experimental equipment enabled simultaneous acquisition of up to six fibre optic gauges. These were packaged in mountings compatible with those of the commercially available electrical (Kulite) pressure sensors. The larger HKM-375 type mounting comprised a threaded steel body and the smaller XCQ-080 style mounting was a $25 \mathrm{~mm}$ long, $2 \mathrm{~mm}$ outside diameter stainless steel tube with the sensor face flush with the tube end (figure 8). The $1 \mathrm{~mm}$ square silicon end face of the sensor body can be seen at the centre of the smaller mounting. If required, sensors can also be mounted directly on a model surface, offering a higher packing density and flexibility of installation.

\section{Experimental test arrangements}

Data on scaling of blast waves in the air are available for spherical and hemispherical explosions. The particular test arrangement used for comparison tests between Kulite and optical sensors was designed to accurately reproduce conditions of a hemispherical blast. This arrangement was chosen because blasts from hemispherical charges on a semi-infinite plane have been widely studied analytically and experimentally, and a large volume of work on the properties of such blast waves at different scales has been published [1].

The small scale experiments were performed in an explosion room at the University of Sheffield CEDUS explosive and hazards testing laboratory, at Buxton. Hemispherical explosive charges between $30 \mathrm{~g}$ and $80 \mathrm{~g}$ of PE4 were initiated at the centre of a $2 \times 2 \mathrm{~m}$ plate made of $12 \mathrm{~mm}$ thick steel as shown in figure 9. In order to allow for unavoidable plastic damage to the plate in the vicinity of a charge, a $15 \mathrm{~cm}$ diameter disk was removed from the centre of the plate, so that the charges could be placed on $15 \mathrm{~cm}$ diameter sacrificial anvils made of $20 \mathrm{~mm}$ thick mild steel. By mismatching the acoustic properties of the anvils and the rest of the structure, as well as using a thin layer of viscous decoupler, the amount of vibration transferred to the test models was significantly reduced. The main plate was welded onto a frame 
made of $80 \mathrm{~mm}$ box section and supported at four corners. The plate centre, where the highest loading occurred, was supported by a separate structure, as shown in Figure 9(c). The main anvil underlying the sacrificial anvil was a $60 \mathrm{~cm}$ diameter steel disc $80 \mathrm{~mm}$ thick, which was bolted to the main plate and supported by a piece of steel tube with an access hole for the centrally placed detonator.

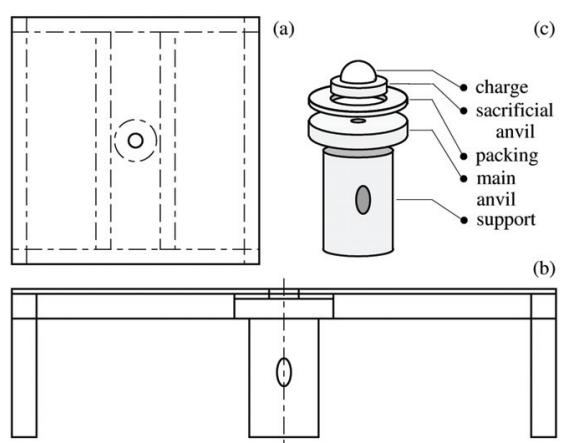

Figure 9. Test arena for producing hemispherical air blasts. (a) Top view (b) side view and (c) the structure of the central support.

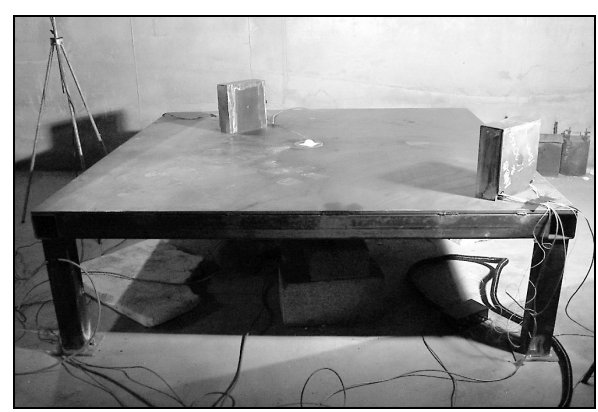

Figure 10. Experimental setup with two rectangular test models.

Two identical test models, hollow rectangular steel boxes $260 \times 270 \times 100 \mathrm{~mm}$, were prepared for the experiments. Both models were spot-welded on the plate diagonals at distances from the centre of the charge to the face of the model of $0.654 \mathrm{~m}$ (model A) and $1.010 \mathrm{~m}$ (model B) respectively (figure 10). Since structure A was situated relatively close to the blast source, it was instrumented with electrical sensors only, to ensure that the optical sensors were not exposed to peak overpressures outside their design range. Electrical gauges \#1 and \#6 were mounted at the centre of front and top faces (figure 11). Model B contained 6 optical sensors and two comparison Kulite sensors.
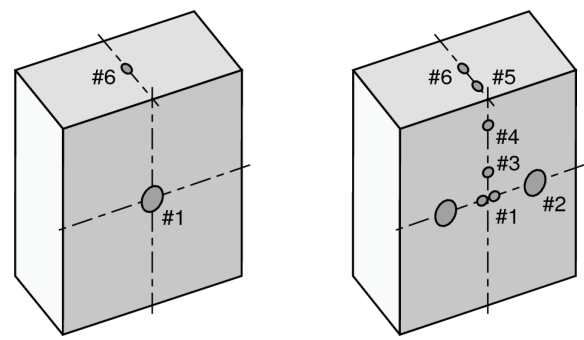

Figure 11. Sensor positions for test models A (left) and B (right). Larger circles correspond to Kulite HKM-375 style mountings, smaller circles to XCQ-080 style mountings.

The fibre optic sensors were arranged (figure 11) to track the propagation of the blast wave from the centre of front face (position \#1) to the centre of top face (position \#6). In order to collect sensor comparison data, readings of the fibre-optic sensors in positions \#1 and \#2 were duplicated by symmetrically placed Kulite gauges. An additional piezoelectric gauge (and optionally an optical gauge) was mounted flush to the test arena main plate at the free diagonal. This side-on gauge, placed $783 \mathrm{~mm}$ away from the arena centre, was used to gather control data free from any diffraction phenomena.

\section{Results}

Over 40 tests were made with explosive charges ranging from 30 to $80 \mathrm{~g}$ of PE4. Although a full review and analysis is beyond the scope of this paper, we present data to indicate the performance of the optical sensors and to illustrate typical features of the blast waves reflected at the front face of a test structure. The pressure-time histories shown in figure 10 were simultaneously recorded by optical and electrical gauges, situated symmetrically on the front face of the test model B, $2.5 \mathrm{~mm}$ either side of the vertical centre line. Figure 12 shows results from the smallest $(30 \mathrm{~g})$ and largest $(80 \mathrm{~g})$ charges respectively. The rise times of 
the optical sensors (10 to $90 \%$ ) were measured to lie between 2 and $4 \mu$ s, whereas typical rise times of the electrical sensors were found to be over $4 \mu$ s both for HKM-375 (range 70 bar, resonant frequency 720 $\mathrm{kHz}$ ) and XCQ-080 (range $35 \mathrm{bar}$, resonant frequency $700 \mathrm{kHz}$ ). Although a shock wave closely approximates to a step change in pressure, the rise times recorded will include transit time across the diaphragm unless the incident shock is accurately normal to the sensor. This is particularly important for gauges situated near the corners and on the top surface of the test models, where significant perturbation of the shock is expected. The small diaphragm diameters of the optical sensors are advantageous in minimising the spatial averaging. Due to the small, low-mass diaphragms, the optical gauges excelled at low acceleration cross-sensitivity. This is evident on comparing the two sensor responses in figure 12; the electrical gauge shows a significant oscillatory component (e.g. between 1.6 and $1.8 \mathrm{~ms}$ in the left-hand plot for a $30 \mathrm{~g}$ charge) due to cantilever vibration of the test structure, which is absent from the optical signal. From the practical point of view this property of the optical sensors ensured more accurate measurements of the positive phase duration of the blast wave as well as the positive impulse. Combined with the lower rise times, we expect that more detailed features of the blast wave can be investigated using the optical data.
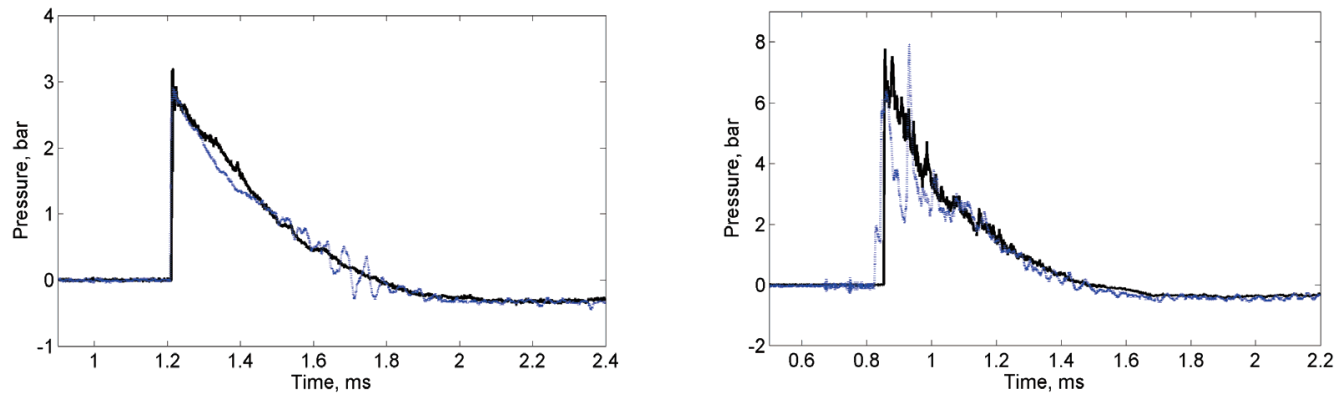

Figure 12. Reflected overpressures recorded at the front face of test structure B using optical (solid) and electrical (dotted) sensors. The distance from the centre of the explosive was constant at $1.010 \mathrm{~m}$; the PE4 explosive charge was $30 \mathrm{~g}$ (left) and $80 \mathrm{~g}$ (right).

The maximum operating pressures of the current optical gauges (approximately 10 bar) limited their experimental use, although they proved to be considerably more robust than might be expected from their size. Typical resolutions varied between $18 \mathrm{mb}$ to $3 \mathrm{mb}$ or range-to-resolution ratios from 550 to 3300 . Although these fall below the performance of the much more highly developed commercial piezoresistive and piezoelectric sensors, the optical sensors' range-to-resolution is sufficient to reveal significant new features of the pressure-time behaviour such as clearing around the boundaries of the test models. Higher operating pressures are possible by using thicker diaphragms, while maintaining the range-to-resolution ratio. Three sensors were irreparably damaged during the trials: two of them were optical, the third was electrical. Most of the optical sensors survived several blasts, and showed no significant change in calibration. Laboratory measurements on the optical sensors over a range 20 to $65^{\circ} \mathrm{C}$ gave temperature sensitivities of typically $0.01 \mathrm{bar} \mathrm{K}^{-1}$. However, on the timescales involved in these tests, no significant thermal cross-sensitivity was observed.

\section{Conclusions}

Favourable performance of the optical sensors has been established against electrical sensors in a series of experimental tests measuring blast waves from small explosive charges. The optical sensors proved to be fast-response, with rise times less than $4 \mu \mathrm{s}$, and were robust enough to survive repeated shocks of up to 8 bar. A particularly useful feature of these sensors is low cross-sensitivity to acceleration, which is crucial to any measurements involving flows around structures. Their extremely small sensing area (50 to $100 \mu \mathrm{m}$ diameter) is a major factor in improving the quality of data, since diaphragms on this scale are less susceptible to spatial averaging of transient shocks present in the flow around an obstacle. Further analysis is progressing to investigate clearing effects at the model edges and corners, and to provide data to test scaling laws against existing results from larger scale experiments. 
Acknowledgements: We acknowledge support from the UK Engineering \& Physical Sciences Research Council and Dstl Fort Halstead through the MoD Joint Grants Scheme. W.N. MacPherson is supported by an EPSRC Advanced Research Fellowship.

\section{References}

[1] Kinney G F and Graham K J 1985 Explosive Shocks in Air, (New York: Springer-Verlag)

[2] Smith P D and Hetherington J G 1994 Blast and Ballistic Loading of Structures (Oxford: Butterworth Heinemann)

[3] Hyde D W 1991 Conventional weapons program (ConWep), US Army Waterways Experimental Station, Vicksburg USA

[4] Technical manual TM 5-855-1 1986 Fundamentals of protective design for conventional weapons Structural Laboratory, Waterways Experimental Station, for the Department of the Army, US Army Corps of Engineers

[5] Tohyama O, Kohashi M, Sugihara M and Itoh H 1998 A fiber-optic pressure microsensor for biomedical applications Sensors and Actuators A-Physical 66 150-154

[6] Zhou J, Dasgupta S, Kobayashi H, Wolff J M, Jackson H E and Boyd J T 2001 Optically interrogated MEMS pressure sensors for propulsion applications Opt. Eng. 40 598-604

[7] Abeysinghe D C, Dasgupta S, Jackson H E, Boyd J T 2002 Novel MEMS pressure and temperature sensors fabricated on optical fibers J. Micromech. and Microeng. $12229-235$

[8] Yu B, Kim D W, Deng J, Xiao H and Wang A 2003 Fiber Fabry-Perot sensors for detection of partial discharges in power transformers Applied Optics 42 3241-3250

[9] Xu J, Pickrell G, Wang X, Peng W, Cooper K and Wang A 2005 A novel temperature-insensitive optical fiber pressure sensor for harsh environments IEEE Phot. Tech. Letters 17 870-872

[10] Cibula E and Đonlagić D 2005 Miniature fiber-optic pressure sensor with a polymer diaphragm Applied Optics $\mathbf{4 4}$ 2736-2744

[11] Totsu K, Haga Y and Esashi M 2005 Ultra-miniature fiber-optic pressure sensor using white light interferometry J. Micromech. and Microeng. 15 71-75

[12] MacPherson W N, Kilpatrick J M, Barton J S and Jones J D C 1999 Miniature fiber optic pressure sensor for turbomachinery applications Rev. Sci. Inst. 70 1868-74

[13] Gander M J, MacPherson W N, Barton J S, Reuben R L, Jones J D C, Stevens R, Chana K S, Anderson S J and Jones T V 2003 Embedded micromachined fiber-optic Fabry-Perot pressure sensors in aerodynamics applications IEEE Sensors $J .3$ 102- 7

[14] Laermer F and Schilp A 1996, Method for anisotropic plasma etching of substrates, US Patent $5,498,312$

[15] MacPherson W N, Kidd S R, Barton J S and Jones J D C 1997 Phase demodulation in optical fibre Fabry-Pérot sensors with inexact phase steps IEE Proc. Optoelectronics 144, 130-133 


\section{List of Figures}

Figure 1. Principle of operation of sensors and optical interrogation system.

Figure 2. Scanning electron microscope cross-section of a portion of the device wafer $380 \mu \mathrm{m}$ thick, showing the fibre entry hole of diameter $128 \mu \mathrm{m}$ above the $50 \mu \mathrm{m}$ diameter sensing cavity.

Figure 3. SEM image of the $\mathrm{SiO}_{2}$ membrane covering one of the $50 \mu \mathrm{m}$ diameter apertures, showing the internal silicon land surrounding the hole at the bottom of the fibre channel.

Figure 4. (a) Illustration of sensor body. (b) Photograph of assembled sensor.

(c) Interference fringes produced by illuminating a sensor with a broadband source.

Figure 5. Pressure cycling and calibration. Top: electrical reference sensor; Middle: optical sensor phase Bottom: calibration curve

Figure 6. Mounted optical fibre sensors: (left) HKM-375 style mounting; (right) XQC-080 style mounting Figure 7. Test arena for producing hemispherical air blasts. (a) Top view (b) side view and (c) the structure of the central support.

Figure 8. Experimental setup with two rectangular test models.

Figure 9. Sensor positions for test models A (left) and B (right). Larger circles correspond to Kulite HKM-375 style mountings, smaller circles to XQC-080 style mountings.

Figure 10. Reflected overpressures recorded at the front face of test structure $B$ using optical (solid) and electrical (dotted) sensors. The distance from the centre of the explosive was constant at $1.010 \mathrm{~m}$; the PE4 explosive charge was $30 \mathrm{~g}$ (left) and $80 \mathrm{~g}$ (right). 